\title{
Application of Hyperthermia for Cancer Treatment: Synthesis and Characterization of Magnetic Nanoparticles and their internalization on Tumor Cell Lines*
}

\author{
Catarina I. P. Chaparro, Liliana R. Loureiro, Manuel Almeida Valente, Paula A. Videira, João Paulo \\ Borges and Paula I. P. Soares
}

\begin{abstract}
Truncated sialylated $O$-glycans, such as cellsurface carbohydrate antigen sialyl-Tn (STn) are overexpressed by several cancer types, but not by the respective normal tissues. STn expression is associated with oncogenesis and metastatic ability of cancer cells, with reduced overall survival and lack of response to chemotherapy. Advances in nanomedicine have resulted in rapid development of biocompatible superparamagnetic iron oxide nanoparticles (SPIONs) with considerable potential in cancer treatment. Therefore, in this study SPIONs coated with oleic acid (OA) or dimercaptosuccinic acid (DMSA) were developed and characterized for internalization in two breast cancer cell lines: cell line expressing the STn antigen and the corresponding control. SPIONs with an average diameter of $8 \mathrm{~nm}$ showed superparamagnetic behavior and high potential to be used as magnetic hyperthermia agents. OA and DMSA coating provided high stability of SPIONs in physiological conditions while not changing their main properties. NPs internalization studies showed a higher accumulation of DMSA coated NPs in the breast cancer MDAMB-231 WT cell line. In MDA-MB-231 cell line expressing STn both coated NPs showed a similar accumulation. Therefore, STn antigen can act as a receptor capable of detecting and covalently bind to the molecules present on NPs surface and induce their cellular uptake by endocytosis.
\end{abstract}

\section{INTRODUCTION}

Breast cancer is the most common malignancy in women throughout the world, accounting for more than $25 \%$ of all new cancer diagnoses in 2012, and despite significant improvements in treatment, for 522,000 deaths [1]. The cellsurface carbohydrate antigen STn, which is not observed in normal healthy tissues, is overexpressed in 30-40 \% of early stage cases of breast cancer and so it is considered an excellent candidate to target initial phases of malignancy [2]. STn expression modulate a malignant phenotype inducing a more aggressive cell behavior in breast carcinoma cells. These facts clearly indicate the need to improve existing cancer treatments and to develop new therapeutic strategies. Nanomedicine, the application of nanotechnology to medicine, is a promising approach for specific cancer cell targeting, diagnosis, and treatment [3]. Among the nanotechnology tools investigated

*Research supported by National Funds through FCT - Portuguese Foundation for Science and Technology, Reference UID/CTM/50025/2013, FEDER funds through the COMPETE 2020 Program under the project number POCI-01-0145-FEDER-007688. This work was also funded by the Scientific merit prize Santander-Totta - Lisbon New University - "Antibody engineering for breast cancer therapy" 2013. Catarina I. P. Chaparro also acknowledges the financial support from Liga Portuguesa Contra o Cancro (LPCC)/Pfizer 2017. for biomedical applications SPIONs have received a lot of attention in the last decades because of their outstanding magnetic properties, namely superparamagnetism. In particularly they are used as contrast agents for magnetic resonance imaging (MRI), as cell labeling agents for cell tracking, as drug delivery carriers and to induce magnetic hyperthermia [4,5]. Recently, hybrid NPs formulations with SPION cores modified with exterior coatings and functional probes have been created for their ability to enhance negative contrast in alternative imaging techniques in addition to MRI [6].

Hyperthermia therapy, an adjuvant medical treatment using global or local temperature elevation in tumor tissues, has been successfully applied alone or in combination with radiation and chemotherapy as a cancer treatment tool. This treatment is based on the higher sensitivity of cancer cells, which exhibit compact, disorganized and permeable vascular networks and poor blood flow and retain heat better than viable cells, therefore decreasing the side effects in the normal tissues [7]. In the last few years, research has focused on the injection of magnetic fluids directly into the tumor body, or into an artery supplying the tumor. The method relies on the theory that after localization of SPIONs near the cancer site using magnetic targeting, an alternating magnetic field (AMF) is applied to induce heat of about $42-45^{\circ} \mathrm{C}$ for initiating apoptosis in cancer cells. This temperature increase can be controlled by manipulating the corresponding magnetic properties of NPs and the applied AMF [8].

In this work we studied the influence of OA and DMSA on the stability of colloidal solutions containing iron oxide $\left(\mathrm{Fe}_{3} \mathrm{O}_{4}\right) \mathrm{NPs}$ for further cellular uptake studies in breast cancer cells. This surface stabilization protects the magnetic core against oxidation and avoid the generation of reactive oxygen species (ROS) upon exposure of cells to SPIONs, preventing cytotoxicity effects [9]. The focus of this study was to design and develop nanostructures based on iron oxide nanoparticles stable in physiological conditions and able to generate heat under the application of an external magnetic field. The interaction of OA and DMSA $\mathrm{Fe}_{3} \mathrm{O}_{4}$ NPs was evaluated through cytotoxicity assays and cellular internalization of

Catarina I. P. Chaparro, João Paulo Borges and Paula I. P. Soares are with the CENIMAT/i3N, FCT-UNL, Caparica, 2829-516 Portugal (corresponding author to provide phone: +351 212948564; fax: +351 212957810; e-mail: c.chaparro@campus.fct.unl.pt).

Liliana R. Loureiro and Paula A. Videira are with UCIBIO, FCT-UNL, Caparica, Lisbon 2829-516 Portugal.

Manuel Almeida Valente is with I3N, University of Aveiro, Aveiro 3810193 Portugal. 
these nanostructures in two breast cancer cell lines: MDAMB-231 WT and STn-expressing MDA-MB-231.

\section{MATERIALS \& METHODS}

SPIONs synthesis was performed through chemical coprecipitation which gives a careful control of the reaction parameters, like size and shape [10]. To obtain stable nanoparticles, OA and DMSA were used as coating molecules since they allow high stability of the nanoparticles in aqueous media and have free ligand groups for further biomolecule conjugation. OA was added to the NPs suspension as a percentage of the NPs mass. The mixture reacted during 4 hours in ultrasound bath. These NPs will be referenced as OA $\mathrm{Fe}_{3} \mathrm{O}_{4}$ NPs. Before coating the NPs with DMSA, the $\mathrm{pH}$ of the suspension was adjusted to $\mathrm{pH} 3$ with nitric acid $65 \%$ under magnetic agitation. DMSA was added immediately at a $4 \%$ ratio of $[\mathrm{DMSA}] /\left[\mathrm{Fe}^{2+}\right][11]$. DMSA was dissolved in $10 \mathrm{~mL}$ of deionized water and the $\mathrm{pH}$ adjusted to 5.5 with $0.1 \mathrm{M}$ $\mathrm{NaOH}$ solution under vigorous stirring. The reaction of DMSA with NPs was performed in ultrasound bath for 4 hours. To characterize the stabilized SPIONs the following techniques were used: X-ray Diffraction (XRD) and Fourier Transform Infrared (FTIR) spectrometry to evaluate the structural and physical properties, UV-Vis spectrophotometry to evaluate the colloidal stability, Transmission electron microscopy (TEM) to evaluate the morphology, Vibrating sample magnetomer (VSM) to verify the magnetic properties and Dynamic light scattering (DLS) to measure the particles sizes in solution. Magnetic hyperthermia measurements were performed using a DM100 series from Nb Nanoscale Biomagnetics apparatus. Cell viability was determined using the MTS assay colorimetric method that measure mitochondrial activity. Cellular targeting and uptake of SPIONs were assessed by qualitative and quantitative studies that shows the effect of SPIONs molecular coatings in cellular uptake. For qualitative studies, Prussian Blue Staining technique was performed [12]. This method reveals the visual uptake of nanoparticles through bright field microscopy while 1,10-phenantroline colorimetric method was used to evaluate SPIONs internalization in a quantitative way indicating the iron uptake by cells.

\section{RESULTS \& DISCUSSION}

\section{A. Iron oxide nanoparticles characterization}

One of the biggest concerns related to the use of nanostructures in biomedical applications include their stability in aqueous solution as well as their biocompatibility in cells/tissues. In this work, SPIONs were surface modified using OA and DMSA as stabilizers agents. SPIONs were synthesized by chemical co-precipitation with an average core diameter of $8 \mathrm{~nm}$ as showed in Fig. 1. Physicochemical characterization allowed the confirmation and identification of crystal structure preservation and the main chemical bonds formed after the $\mathrm{Fe}_{3} \mathrm{O}_{4}$ NPs surface. For a $64 \%$ OA concentration, $\mathrm{Fe}_{3} \mathrm{O}_{4}$ NPs have a hydrodynamic diameter of $147.3 \pm 5.5 \mathrm{~nm}$ and DMSA (4\%) coated $\mathrm{Fe}_{3} \mathrm{O}_{4}$ NPs obtained a $57.1 \pm 1.7 \mathrm{~nm}$ diameter, with a polydispersity index (PDI) of $0.25 \pm 0.02$ and $0.20 \pm 0.01$, respectively.

Thus, we concluded that both coating molecules provided high stability of SPIONs in physiological conditions while not changing their main properties although the nanoparticles hydrodynamic diameter is significantly higher for those stabilized with OA compared to DMSA. Table I summarizes the properties of the studied samples. Naked iron oxide NPs exhibit a magnetic saturation $\left(M_{\mathrm{S}}\right)$ of around $57 \mathrm{emu} . \mathrm{g}^{-1} \mathrm{Fe}$ at $320 \mathrm{~K}$. While DMSA does not change the magnetic saturation of the NPs, OA reduces the $M_{S}$ of the NPs to $45 \mathrm{emu} . \mathrm{g}^{-1}$. This suggests that the surfactant is reducing the magnetic moments at the surface of the NPs probably due to the diamagnetic contribution of OA volume. The surface atoms to volume ratio in NPs increases and consequently reduces the saturation magnetization [13]. Fig. 2 (A) represents the hysteresis loops of naked NPs and $\mathrm{Fe}_{3} \mathrm{O}_{4}$ NPs coated with $64 \%$ OA and $4 \%$ DMSA, measured at $320 \mathrm{~K}$. For both cases it is evident the absence of coercivity and remanence at $320 \mathrm{~K}$, which confirms the superparamagnetic behavior of all samples above the blocking temperature. In all cases, the magnetic domains are not separate by domain walls (multidomain state). Instead, each particle represents a single magnetic domain [14].
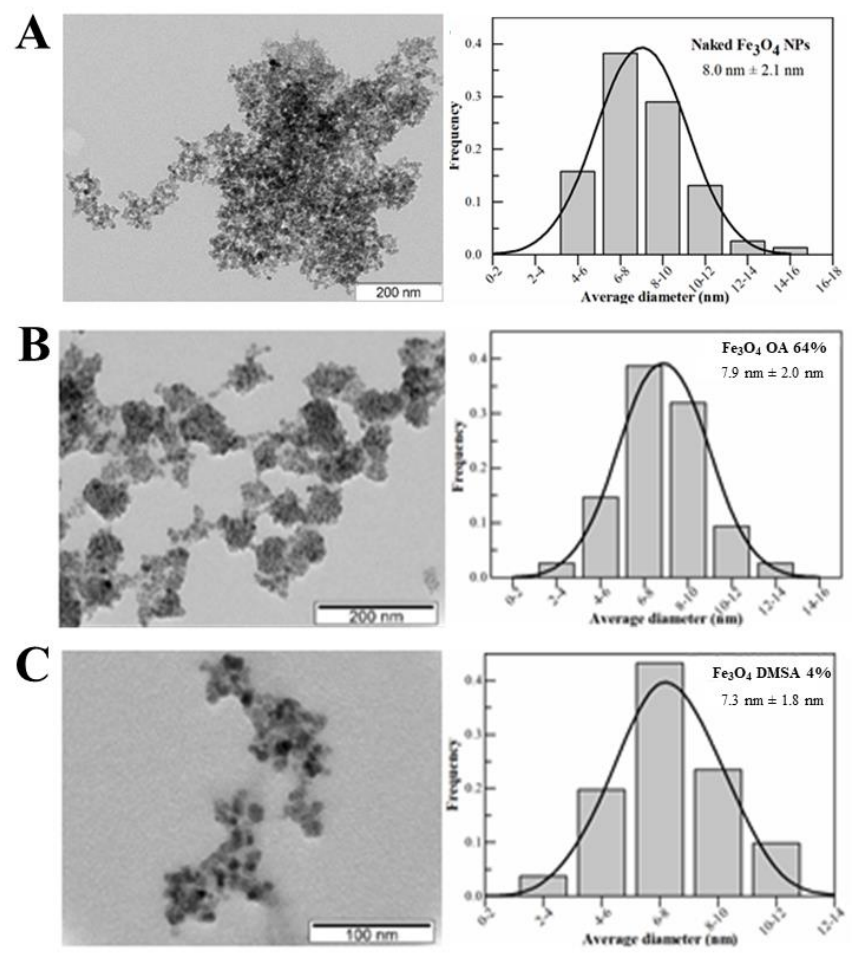

Figure 1. TEM images of $(A)$ naked $(8.0 \pm 2.1 \mathrm{~nm}),(\mathrm{B}) \mathrm{OA}(7.9 \pm 2.0 \mathrm{~nm})$ and $(\mathrm{C})$ DMSA $(7.3 \pm 1.8 \mathrm{~nm}) \mathrm{Fe}_{3} \mathrm{O}_{4}$ NPs.

TABLE I. PROPERTIES OF OA AND DMSA FE $\mathrm{O}_{3}$ NPS. AVERAGE SIZE AND AVERAGE DIAMETER OBTAINED FROM XRD PATTERN, DLS AND TEM IMAGE, RESPECTIVELY. MAGNETIC PROPERTIES ARE PRESENTED BY MAGNETIC SATURATION $\left(M_{S}\right)$ VALUES.

\begin{tabular}{|c|c|c|c|}
\hline \multicolumn{2}{|c|}{ Samples } & $\begin{array}{c}\mathrm{OA} \mathrm{Fe}_{3} \mathrm{O}_{4} \\
\mathrm{NPs}\end{array}$ & $\begin{array}{c}\text { DMSA } \mathrm{Fe}_{3} \mathrm{O}_{4} \\
\text { NPs }\end{array}$ \\
\hline \multicolumn{2}{|c|}{ Average crystallite size (XRD) (nm) } & 9.75 & 9.75 \\
\hline \multicolumn{2}{|c|}{ Average diameter (TEM) (nm) } & $7.9 \pm 2.0$ & $7.3 \pm 1.8$ \\
\hline \multirow{2}{*}{$\begin{array}{l}\text { Average diameter } \\
\text { (DLS) }\end{array}$} & $\mathrm{D}_{\mathrm{h}}(\mathrm{nm})$ & $147.3 \pm 5.5$ & $57.1 \pm 1.7$ \\
\hline & PDI & $0.25 \pm 0.02$ & $0.20 \pm 0.01$ \\
\hline \multicolumn{2}{|c|}{$M_{S}\left(\mathrm{emu} \cdot \mathrm{g}^{-1} \mathrm{Fe}\right)$} & 45 & 57 \\
\hline
\end{tabular}

To access the influence of DMSA and OA molecules in the heating generation ability of SPIONs, magnetic hyperthermia 
measurements were performed. Fig. 2 (B) represents the temperature variation obtained from magnetic hyperthermia test as a function of iron concentration for both OA and DMSA $\mathrm{Fe}_{3} \mathrm{O}_{4} \mathrm{NPs}$. For OA $\mathrm{Fe}_{3} \mathrm{O}_{4} \mathrm{NPs}$, we can observe a tendency of the heating ability of the NPs to stabilize for high iron concentrations. However, an exponential temperature increase is observed for DMSA $\mathrm{Fe}_{3} \mathrm{O}_{4}$ NPs. For the same iron concentration, these NPs can increase their temperature about $2 \mathrm{x}$ than $\mathrm{OA} \mathrm{Fe}_{3} \mathrm{O}_{4}$ NPs. From magnetic hyperthermia measurements it is possible to obtain not only the temperature variation but also to calculate the heating efficiency of the magnetic material. This parameter can be determined using the specific absorption rate (SAR). SAR values (Fig. 2 (C)) remained constant with increasing iron concentrations for both coated NPs. However, SAR values are found to be twice as low for $\mathrm{OA} \mathrm{Fe} \mathrm{O}_{4} \mathrm{NPs}$ compared to DMSA Fe $\mathrm{O}_{4} \mathrm{NPs}$. These results can be explained by the relaxation modes of magnetic NPs. When a magnetic material is submitted to an alternating current (AC) magnetic field, exhibits both Brownian and Néel relaxations. When the NPs are in suspension both relaxation paths are present. Brownian relaxation occurs due to the ability of the particles to freely rotate. In this case, the bilayer formed around the iron oxide cores entrap the NPs thus eliminating their Brownian movements. Therefore, for $\mathrm{OA} \mathrm{Fe}_{3} \mathrm{O}_{4} \mathrm{NPs}$ the heat generation can only be attributed to Néel relaxation [15]. This could additionally explain the low $\mathrm{M}_{\mathrm{S}}$ observed in these samples.

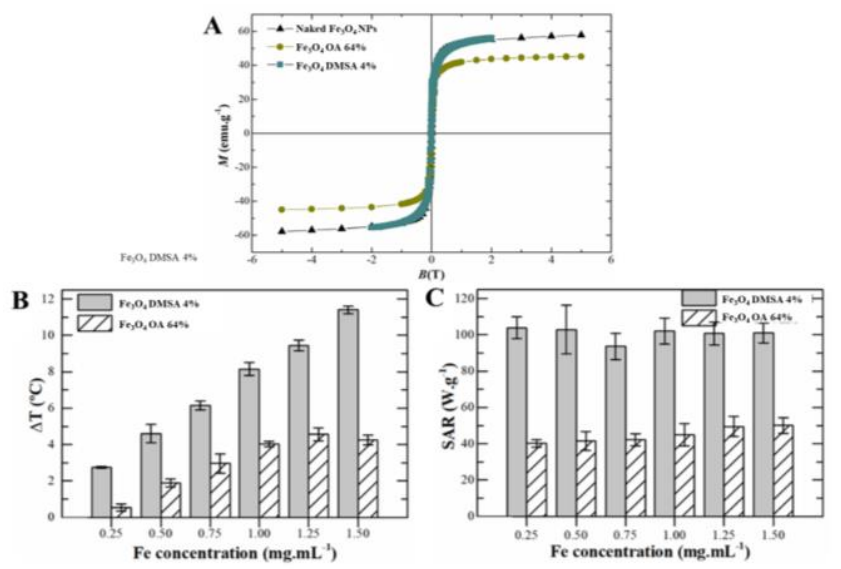

Figure 2. Magnetic characterization (A) of naked, OA and DMSA $\mathrm{Fe}_{3} \mathrm{O}_{4}$ NPs. (B) Generated temperature of OA and DMSA $\mathrm{Fe}_{3} \mathrm{O}_{4}$ NPs during 10 min of AC magnetic field application with an intensity of $24 \mathrm{kAm}^{-1}$ and $418.5 \mathrm{kHz}$ of frequency as a function of iron concentration. (C) Comparison of SAR values of $\mathrm{OA}$ and DMSA $\mathrm{Fe}_{3} \mathrm{O}_{4} \mathrm{NPs}$ in water. The results are expressed as the average \pm standard deviation for three independent experiments.

\section{B. Nanoparticles uptake and internalization in breast cancer cells}

A moderate toxicity is observed for high iron concentrations of around 1.0 and $2.5 \mathrm{mg} \cdot \mathrm{mL}^{-1}$ for $\mathrm{OA}$ and DMSA $\mathrm{Fe}_{3} \mathrm{O}_{4} \mathrm{NPs}$, respectively (Fig. 3). These results provide important input to achieve a compromise between the use of $\mathrm{OA}$ and DMSA as SPIONs stabilizers and its impact on magnetic hyperthermia.

Qualitative and quantitative internalization studies of $\mathrm{OA}$ and DMSA $\mathrm{Fe}_{3} \mathrm{O}_{4}$ NPs in MDA-MB-231 WT and MDA-MB231 STn cell lines were performed (Fig. 4). For the qualitative internalization study of $\mathrm{Fe}_{3} \mathrm{O}_{4} \mathrm{NPs}$, Prussian Blue Staining technique was performed in which uptake of NPs can be observed inside the cells [16]. Fig. 4 (A) shows that increasing the concentration of both NPs lead to a corresponding increase of their cellular internalization in both cell lines.
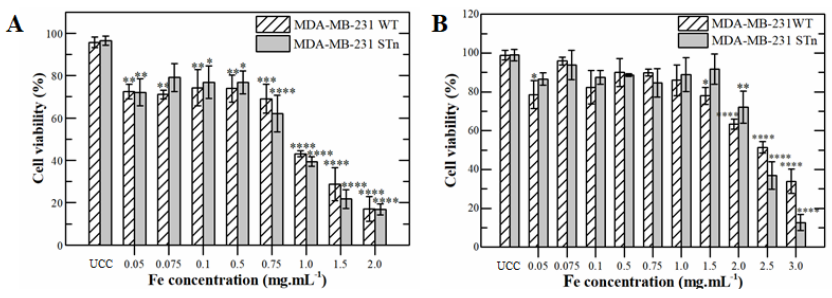

Figure 3. MDA-MB-231 WT and MDA-MB-231 STn cell lines viability after $24 \mathrm{~h}$ exposition to (A) $\mathrm{OA} \mathrm{Fe}_{3} \mathrm{O}_{4} \mathrm{NPs}$ and (B) DMSA Fe $\mathrm{O}_{4} \mathrm{NPs}$.

Results are expressed as average \pm standard deviation for at least three independent experiments. ${ }^{*} \mathrm{p}<0.05,{ }^{* *} \mathrm{p}<0.005,{ }^{* * * *} \mathrm{p}<0.0001$ compared to untreated control cells (UCC).
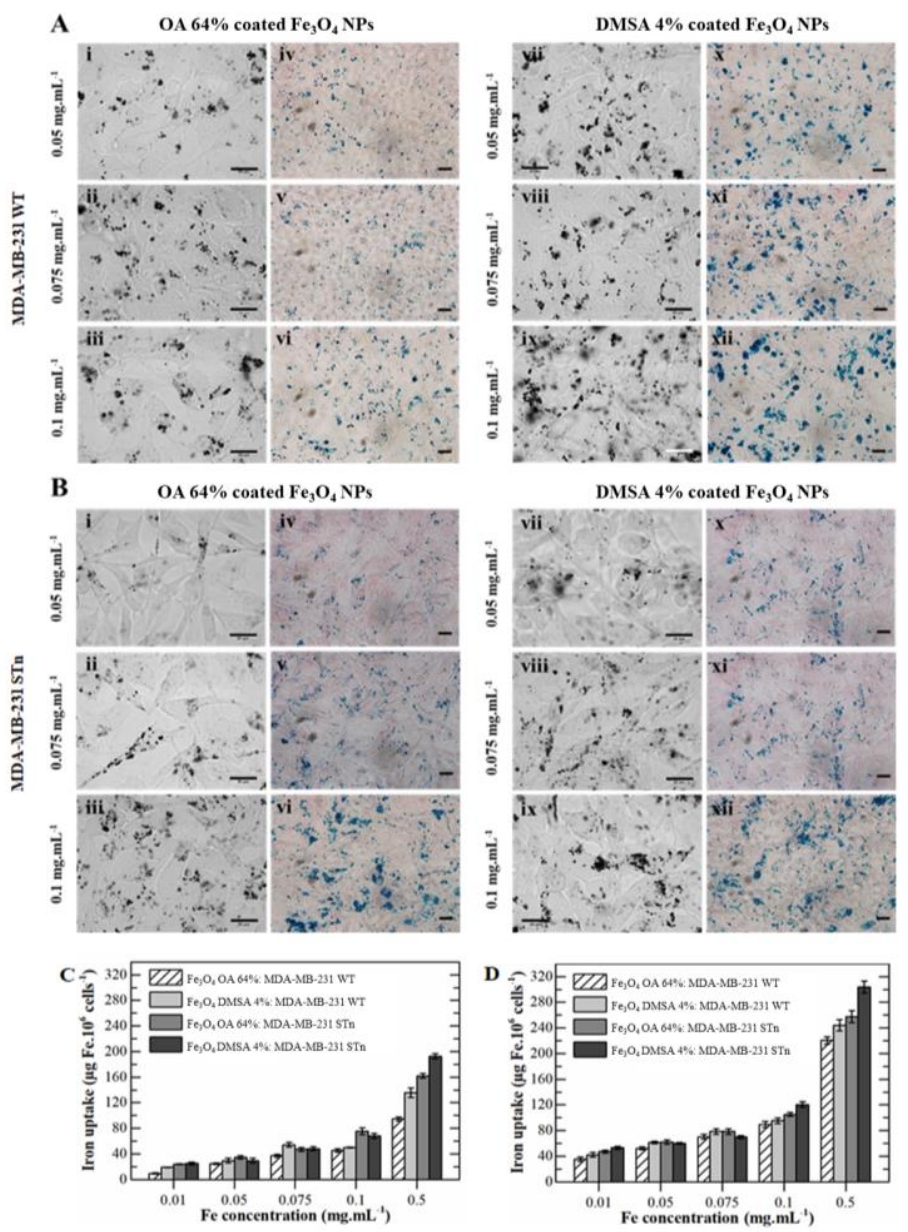

Figure 4. Bright field microscopy visualization of MDA-MB-231 WT (A) and MDA-MB-231 STn (B) cells incubated with OA and DMSA Fe $\mathrm{O}_{4} \mathrm{NPs}^{-}$ at iron concentrations ranging from $0.05 \mathrm{mg} \cdot \mathrm{mL}^{-1}$ to $0.1 \mathrm{mg} \cdot \mathrm{mL}^{-1}$. The blue color inside the cells represents the NPs uptake by contrast between the red and pink colors from the nuclei and cytoplasm staining, respectively. Scale bar represents $20 \mu \mathrm{m}$. (C, D) Intracellular iron content quantification by

1,10-phenanthroline colorimetric method. Iron uptake is expressed as weight of iron per $1 \times 10^{6}$ cells and measured after $24 \mathrm{~h}(\mathrm{C})$ and $48 \mathrm{~h}(\mathrm{D})$ of $\mathrm{OA}$ and DMSA $\mathrm{Fe}_{3} \mathrm{O}_{4}$ NPs incubation in MDA-MB-231 WT and MDA-

MB-231 STn cell lines. Results are expressed as average \pm standard deviation for at least three independent experiments. 
However, this increase is more significant in MDA-MB231 WT cells. Comparing OA and DMSA Fe $\mathrm{O}_{4} \mathrm{NPs}$, it was particularly observed an increased internalization of DMSA $\mathrm{Fe}_{3} \mathrm{O}_{4} \mathrm{NPs}$ due to the larger clusters inside both MDA-MB-231 WT and STn cells (Figure 4 - vii to xii). In several cases it has been shown that a simple DMSA coating can improve uptake efficiency by three orders of magnitude, presumably by engendering the NPs with an anionic charge and resulting in nonspecific adsorption to the cell surface followed by endocytosis into the cell [17]. From Fig. 4 (B), which shows the internalization study of the two types of NPs in the MDAMB-231 STn cell line, two obvious conclusions can be drawn: (i), NPs internalization increases proportionally to the increase of OA ( $\mathrm{i}$ to vi) and DMSA $\mathrm{Fe}_{3} \mathrm{O}_{4} \mathrm{NPs}$ (vii to xii) concentrations and (ii), although it is slightly more pronounced in DMSA NPs, both NPs uptake in MDA-MB-231 STn cell line seems to be similar.

This cell line expresses the carbohydrate antigen STn which occurs in approximately $80 \%$ of human cancers [2]. $\mathrm{STn}$ is a short O-glycan terminated by a sialic acid named NAcetylneuraminic acid which can potentially establish a specific binding with the coating molecules of NPs. The carbonyl group $(\mathrm{C}=\mathrm{O})$ of this monosaccharide, may be available for a chemical bond sharing with the functional groups of OA and DMSA molecules A known method of NPs internalization in cells is based on a receptor-mediated approach, by which specific ligands are covalently linked to the coating molecules present on NPs to induce cell membrane receptor recognition followed by subsequent endocytosis [7,17]. Thus, two possible conclusions can be drawn: (i) OA and DMSA NPs internalization in MDA-MB-231 WT cell line is considered as non-specific, since the NPs can cross the cell membrane without any receptor involvement; (ii) the internalization of the same NPs in MDA-MB-231 STn cell line represents a NP-cell binding mediated by a receptor, which in this case is proposed to be the sialic acid present in the STn antigen. Therefore, the cellular internalization of NPs is much more effective in this last case.

After $24 \mathrm{~h}$ (Fig. 4 (C)) and $48 \mathrm{~h}$ (Fig. 4 (D)) of incubation with $\mathrm{OA}$ and DMSA $\mathrm{Fe}_{3} \mathrm{O}_{4} \mathrm{NPs}$ the iron uptake in cells shows a gradual increase, considering that from 0.1 to $0.5 \mathrm{mg} . \mathrm{mL}^{-1}$ an exponential increase can be observed. This effect may be due to larger agglomerates accumulation in cells considering the higher concentration administered. Overall, it is possible to conclude that MDA-MB-231 STn cells can internalize more NPs compared to the WT cell line and that DMSA Fe ${ }_{3} \mathrm{O}_{4}$ NPs have a higher internalization rate than those stabilized with OA. Additionally, the concentration of absorbed iron is almost doubled after $48 \mathrm{~h}$ of incubation with NPs compared to the iron uptake values obtained after $24 \mathrm{~h}$ incubation.

\section{CONCLUSIONS}

We have developed stable $\mathrm{Fe}_{3} \mathrm{O}_{4}$ NPs in solution and for that reason intracellular accumulation of these NPs is an important parameter to consider for biomedical applications. Qualitative and quantitative NPs internalization studies conclude that two types of cellular internalization may occur: (i) non-specific internalization of NPs, or (ii) receptormediated internalization. In summary, the results herein obtained suggest that DMSA coated NPs are the most suitable for cancer treatment using magnetic hyperthermia.
Considering the properties of DMSA coating, we envision the functionalization of the magnetic NPs surface with a monoclonal antibody against STn antigen to provide increased specificity to this system in the targeting of STn expressing cancer cells. In light of this results, it would be interesting to use this coated $\mathrm{Fe}_{3} \mathrm{O}_{4}$ NPs in other biomedical applications, such as contrast agents for MRI.

\section{REFERENCES}

[1] Cancer Research UK, 2014. Worldwide cancer incidence statistics. Available at: http:/www.cancerresearchuk.org/healthprofessional/cancer-statistics/worldwide-cancer/incidence [Accessed December 1, 2018]

[2] L. R. Loureiro, M. A. Carrascal, A. Barbas, J. S. Ramalho, C. Novo, P. Delannoy, et al., Challenges in Antibody Development against Tn and Sialyl-Tn Antigens. Biomolecules. 2015;5:1783-809

[3] Lammers T., Aime S., Hennink WE., Storm G., Kiessling F., Theranostic nanomedicine. AccChem Res. 2011;44: 1029-1038

[4] P. I. P. Soares, I. M. M. Ferreira, R. A. G. B. N. Igreja, C. M. M. Novo and J. P. M. R. Borges, Application of Hyperthermia for Cancer Treatment: Recent Patents Review. Recent patents on anti-cancer drug discovery. 2012;7:64-73

[5] R. J. R. Matos, C. I. P. Chaparro, J. Silva, M. A. Valente, J. P. Borges, P. I. P. Soares, Electrospun composite cellulose acetate/iron oxide nanoparticles nonwoven membranes for magnetic hyperthermia applications. Carbohydrate Polymers. 2018;198:9-16

[6] R. A. Revia and M. Zhang, Magnetite nanoparticles for cancer diagnosis, treatment, and treatment monitoring: recent advances. Mater Today (Kidlington). 2016;19:157-168

[7] C. C. Berry, Progress in functionalization of magnetic nanoparticles for applications in biomedicine. Journal of Physics D: Applied Physics. 2009;42:224003

[8] R. Fernando, J. Downs, D. Maples and A. Ranjan, MRI-guided monitoring of thermal dose and targeted drug delivery for cancer therapy. Pharm Res. 2013;30:2709-17

[9] S. Laurent, D. Forge, M. Port, A. Roch, C. Robic, L. Vander Elst, et al., Magnetic iron oxide nanoparticles: synthesis, stabilization, vectorization, physicochemical characterizations, and biological applications. Chem Rev. 2008;108:2064-110

[10] P. I. Soares, A. M. Alves, L. C. Pereira, J. T. Coutinho, I. M. Ferreira, C. M. Novo, et al., Effects of surfactants on the magnetic properties of iron oxide colloids. J Colloid Interface Sci. 2014;419:46-51

[11] N. Fauconnier, J. N. Pons, J. Roger and A. Bee, Thiolation of Maghemite Nanoparticles by Dimercaptosuccinic Acid. J Colloid Interface Sci. 1997;194:427-3

[12] M. Calero, M. Chiappi, A. Lazaro-Carrillo, M. J. Rodriguez, F. J. Chichon, K. Crosbie-Staunton, et al., Characterization of interaction of magnetic nanoparticles with breast cancer cells. J Nanobiotechnology. 2015;13:16

[13] P. I. Soares, F. Lochte, C. Echeverria, L. C. Pereira, J. T. Coutinho, I. M. Ferreira, et al., Thermal and magnetic properties of iron oxide colloids: influence of surfactants. Nanotechnology. 2015;26:425704

[14] X. Q. Liu, M. D. Kaminski, Y. P. Guan, H. T. Chen, H. Z. Liu and A. J. Rosengart, Preparation and characterization of hydrophobic superparamagnetic magnetite gel. Journal of Magnetism and Magnetic Materials. 2006;306:248-253

[15] P. I. P. Soares, C. A. T. Laia, A. Carvalho, L. C. J. Pereira, J. T. Coutinho, I. M. M. Ferreira, et al., Iron oxide nanoparticles stabilized with a bilayer of oleic acid for magnetic hyperthermia and MRI applications. Applied Surface Science. 2016;383:240-247

[16] S. H. Liao, C. H. Liu, B. P. Bastakoti, N. Suzuki, Y. Chang, Y. Yamauchi, et al., Functionalized magnetic iron oxide/alginate coreshell nanoparticles for targeting hyperthermia. Int $J$ Nanomedicine. 2015;10:3315-27

[17] C. Wilhelm and F. Gazeau, Universal cell labelling with anionic magnetic nanoparticles. Biomaterials. 2008;29:3161-74 